\title{
The Relationship Between Symptoms of Oral Mucosal Diseases and Psychological Status
}

Hye Sook Park

\author{
Department of Dental Technology, Shingu College, Seongnam, Korea
}

Received February 16, 2021

Revised March 8, 2021

Accepted March 9, 2021
Correspondence to:

Hye Sook Park

Department of Dental Technology, Shingu

College, 377 Gwangmyeong-ro, Jungwon-

gu, Seongnam 13174, Korea

Tel: +82-31-740-1575

Fax: +82-31-740-1589

E-mail: hspark@shingu.ac.kr

https://orcid.org/0000-0002-2981-3908

This study was supported by a grant from Industry-Academy Cooperation Foundation of Shingu College.
Purpose: The purpose of this study was to assess the relationship between psychological factors and symptoms of oral mucosal diseases by means of the revised version of the Minnesota Multiphasic Personality Inventory (MMPI-2).

Methods: Three hundred and eighteen college students in Gyeonggi-do completed the MMPI-2 and a questionnaire related to symptoms of oral mucosal diseases and collected data were analyzed by Student's t-test and one way ANOVA.

Results: Subjects who have experienced herpetic stomatitis revealed significantly higher mean values of T-scores than subjects who have not on hypochondriasis (Hs) and hysteria (Hy) clinical scales of the MMPI-2 ( $<<0.05)$. In comparison with subjects who have had recurrent aphthous ulceration, subjects who have not had it manifested significantly higher elevations on the schizophrenia (Sc) clinical scale $(\mathrm{p}<0.05)$. Subjects who have suffered oral lichen planus (OLP) showed significantly higher mean values of T-scores than subjects who have not on paranoia $(\mathrm{Pa})(\mathrm{p}<0.05)$ and hypomania $(\mathrm{Ma})(\mathrm{p}<0.01)$ clinical scales. Mean values of T-scores on Ma clinical scale were significantly higher in subjects having experienced glossodynia than in subjects having not experienced $(\mathrm{p}<0.05)$. Subjects having felt taste disturbance exhibited significantly higher elevations on Hs ( $p<0.0001)$ and Hy $(p<0.01)$ clinical scales compared to subjects having not.

Conclusions: Symptoms of oral mucosal diseases such as herpetic stomatitis, taste disturbance, OLP, and glossodynia were found to be related to Hs, Hy, Pa, Ma clinical scales of the MMPI-2. Therefore, comprehensive psychological evaluations of those oral mucosal diseases may be beneficial in a therapeutic approach.

Key Words: Glossodynia; Minnesota Multiphasic Personality Inventory; Oral mucosal diseases; Psychological factor; Taste disturbance

\section{INTRODUCTION}

There have been research studies attempting to relate psychosocial factors with oral mucosal diseases [1]. Factors implicated in the promotion and exacerbation of recurrent aphthous ulceration (RAU) include infection, nutrition, hormones, psychologic stress, trauma and allergy [2]. The statistically different levels of psychological stress observed between the RAU and control group support the role of stress as a modifier of RAU [3]. Psychological disorders have been considered as possible etiological factors of oral lichen planus (OLP) [4]. The OLP patients had significantly higher stress, anxiety and depression levels when measured by the General Health Questionnaire (GHQ) and the Hospital Anxiety and Depression Scale (HADS) respectively [5]. Recurrent oral herpes is a multifactorial process in which stressful life events and situations seem to be, in certain individuals, a very important factor [1]. Social or emotional 
discomfort acts as a precipitating factor for individual episodes of recurrent herpes labialis [6]. Effects of stress on natural and specific immune responses may predispose the host to more severe Candida infections [7]. Risk factors of oral candidiasis (thrush) include impaired salivary gland function, drugs, dentures, malnutrition, extremes of life, diabetes mellitus, malignancies, and immunosuppressive conditions [8]. Extremes of life predispose to infection because of reduced immunity [9]. It seems that there are two main categories of glossodynia or burning mouth syndrome (BMS), one with a detectable organic etiology and another with a psychologic origin [10]. Both the scores of anxiety, depression in eighty-seven consecutive patients with BMS were higher than those of the control group by Self-rating Anxiety Scale and Self-rating Depression Scale [11]. Taste disturbance has been found associated with local, systemic, and psychological factors [12,13]. State anxiety, perceived stress, depression, and trait anxiety had significant associations with perceived taste disturbance [14]. It has been suggested that halitosis is a symptom related to both somatic and emotional status, and that psychological disorders are strongly associated with reporting the condition in some patients [15,16]. Oho et al. [17] reported a significant correlation between the degree of halitosis and the tendency toward neurosis by using the Cornell Medical Index Health Questionnaire [17].

Although the revised version of the Minnesota Multiphasic Personality Inventory (MMPI-2) is one of the most comprehensive psychological inventories, its use for psychometric assessment of some oral mucosal diseases is relatively uncommon. Hence, the aim of the present study is to assess the relationship between psychological factors and symptoms of oral mucosal diseases by means of MMPI-2.

\section{MATERIALS AND METHODS}

This study is approved by the Institutional Review Board of Shingu College (IRB-2020-101).

\section{Subjects}

Data were collected from three hundred and eighteen college students in Gyeonggi-do, including sixty eight men and two hundred and fifty women. Mean age of the subjects was $20.0 \pm 2.8$ years (Table 1 ).

Data were obtained from October to December of 2020. Visiting a classroom, an examiner explained in detail each item of the questionnaire (Appendix 1) related to symptoms of oral mucosal diseases and asked the students to answer it by self-evaluation and retrieved the completed answers at their classroom. Moreover, a psychologist administered MMPI-2 to the students.

\section{Statistical Analyses}

All the statistical analyses were performed by using IBM SPSS Statistics for Windows, Version 25.0 (IBM Co., Armonk, NY, USA). Student's t-test was used to compare mean values of T-scores on hypochondriasis (Hs), depression (D), hysteria (Hy), psychopathic deviation (Pd), paranoia (Pa), psychasthenia (Pt), schizophrenia (Sc), hypomania (Ma), and social introversion (Si) clinical scales between subjects having experienced symptoms of oral mucosal diseases and subjects having not experienced them. One way ANOVA was implemented to compare mean values of T-scores on Hs, D, Hy, Pd, Pa, Pt, Sc, Ma, and Si clinical scales according to the subjective degree of halitosis. Probability values of $p<0.05$ were accepted as statistically significant.

\section{RESULTS}

In the current study 103 (32.4\%) of subjects had the symptom of thrush, 144 (45.3\%) herpetic stomatitis, 116 (36.5\%) RAU, 50 (15.7\%) OLP, 60 (18.9\%) glossodynia, 22 (6.9\%) taste disturbance, 145 (45.6) halitosis (Table 2).

Subjects who have experienced herpetic stomatitis revealed significantly higher mean values of T-scores than subjects who have not on Hs and Hy clinical scales of the MMPI-2 ( $<<0.05)$. In comparison with subjects who have had RAU, subjects who have not had it manifested

Table 1. Demographics of the subjects

\begin{tabular}{lcc}
\hline Gender & Subject & Age $(\mathrm{y})$ \\
\hline Men & $68(21.4)$ & $20.8 \pm 3.5$ \\
Women & $250(78.6)$ & $19.7 \pm 2.6$ \\
Total & $318(100.0)$ & $20.0 \pm 2.8$ \\
\hline
\end{tabular}

Values are presented as number (\%) or mean \pm standard deviation. 
Table 2. Comparison of mean values of T-scores on $\mathrm{Hs}, \mathrm{D}, \mathrm{Hy}, \mathrm{Pd}, \mathrm{Pa}, \mathrm{Pt}, \mathrm{Sc}, \mathrm{Ma}$, and $\mathrm{Si}$ clinical scales between subjects having experienced symptoms of oral mucosal diseases and subjects having not experienced them $(n=318)$

\begin{tabular}{|c|c|c|c|c|c|c|c|c|c|}
\hline \multirow{2}{*}{$\begin{array}{l}\text { Symptoms of oral } \\
\text { mucosal diseases }\end{array}$} & \multicolumn{9}{|c|}{ Clinical scales of MMPI-2 } \\
\hline & $\mathrm{Hs}$ & D & Hy & $\mathrm{Pd}$ & $\mathrm{Pa}$ & $\mathrm{Pt}$ & Sc & $\mathrm{Ma}$ & $\mathrm{Si}$ \\
\hline Thrush $(n=103)$ & $49.7 \pm 7.4$ & $50 \pm 10.8$ & $48.2 \pm 7.3$ & $43.1 \pm 8.4$ & $48.8 \pm 8.4$ & $49.6 \pm 11.2$ & $45.2 \pm 8.5$ & $48.3 \pm 9.6$ & $50.1 \pm 12.5$ \\
\hline No thrush (n=215) & $49.3 \pm 7.6$ & $49.2 \pm 10.3$ & $48.7 \pm 8.3$ & $43.8 \pm 9.3$ & $48.3 \pm 9.1$ & $49.4 \pm 10.5$ & $45.0 \pm 8.7$ & $48.1 \pm 9.0$ & $48.9 \pm 11.9$ \\
\hline$p$-value & 0.656 & 0.545 & 0.638 & 0.520 & 0.638 & 0.832 & 0.879 & 0.868 & 0.413 \\
\hline Herpes $(n=144)$ & $50.7 \pm 8.4$ & $49.6 \pm 10.4$ & $49.6 \pm 8.5$ & $44.5 \pm 9.2$ & $48.9 \pm 9.8$ & $49.2 \pm 11.5$ & $45.6 \pm 9$ & $48.7 \pm 9.8$ & $48.9 \pm 11.2$ \\
\hline $\begin{array}{l}\text { No herpes } \\
(n=174)\end{array}$ & $48.5 \pm 6.6$ & $49.3 \pm 10.5$ & $47.6 \pm 7.5$ & $42.8 \pm 8.8$ & $48.1 \pm 8.1$ & $49.6 \pm 10.0$ & $44.7 \pm 8.3$ & $47.6 \pm 8.7$ & $49.7 \pm 12.8$ \\
\hline p-value & $0.011 *$ & 0.827 & 0.031 * & 0.103 & 0.422 & 0.747 & 0.313 & 0.287 & 0.581 \\
\hline $\operatorname{RAU}(n=116)$ & $49.2 \pm 7.0$ & $49.2 \pm 10.1$ & $47.7 \pm 6.9$ & $42.4 \pm 8.5$ & $47.3 \pm 8.5$ & $48.1 \pm 9.8$ & $43.8 \pm 7.1$ & $48.6 \pm 9.7$ & $49.4 \pm 11.4$ \\
\hline $\begin{array}{l}\text { No RAU } \\
\qquad(n=202)\end{array}$ & $49.6 \pm 7.9$ & $49.6 \pm 10.6$ & $49.0 \pm 8.6$ & $44.3 \pm 9.3$ & $49.0 \pm 9.0$ & $50.2 \pm 11.1$ & $45.8 \pm 9.3$ & $47.9 \pm 8.9$ & $49.3 \pm 12.5$ \\
\hline p-value & 0.677 & 0.745 & 0.195 & 0.079 & 0.099 & 0.084 & $0.047 *$ & 0.464 & 0.965 \\
\hline OLP $(n=50)$ & $51.3 \pm 8.7$ & $49.1 \pm 11.0$ & $50.0 \pm 8.0$ & $44.7 \pm 8.8$ & $50.8 \pm 11.0$ & $50.2 \pm 12.2$ & $47.0 \pm 10.0$ & $51.3 \pm 10.4$ & $47.8 \pm 11.5$ \\
\hline No OLP $(n=268)$ & $49.1 \pm 7.3$ & $49.5 \pm 11.0$ & $48.2 \pm 8.0$ & $43.4 \pm 9.1$ & $48.0 \pm 8.4$ & $49.3 \pm 10.4$ & $44.7 \pm 8.3$ & $47.5 \pm 8.9$ & $49.6 \pm 12.2$ \\
\hline $\mathrm{p}$-value & 0.062 & 0.819 & 0.157 & 0.348 & 0.036 * & 0.583 & 0.091 & $0.008^{* *}$ & 0.328 \\
\hline $\begin{array}{l}\text { Glossodynia } \\
(n=60)\end{array}$ & $50.8 \pm 8.4$ & $47.8 \pm 9.9$ & $49.9 \pm 8.4$ & $44.1 \pm 8.8$ & $48.9 \pm 8.5$ & $49.1 \pm 11.6$ & $45.0 \pm 8.7$ & $50.4 \pm 9.3$ & $46.6 \pm 12.3$ \\
\hline $\begin{array}{l}\text { No glossodynia } \\
\qquad(n=258)\end{array}$ & $49.2 \pm 7.3$ & $49.8 \pm 10.5$ & $48.2 \pm 7.9$ & $43.5 \pm 9.1$ & $48.3 \pm 9.0$ & $49.5 \pm 10.5$ & $45.1 \pm 8.6$ & $47.6 \pm 9.1$ & $50.0 \pm 12.0$ \\
\hline $\mathrm{p}$-value & 0.136 & 0.178 & 0.150 & 0.643 & 0.636 & 0.765 & 0.949 & $0.032 *$ & 0.050 \\
\hline $\begin{array}{l}\text { Taste disturb- } \\
\text { ance }(n=22)\end{array}$ & $55.7 \pm 10.8$ & $53.1 \pm 12.9$ & $53.0 \pm 10.9$ & $43.3 \pm 10.4$ & $50.8 \pm 8.4$ & $52.6 \pm 14.7$ & $48.1 \pm 12.4$ & $48.9 \pm 11.4$ & $52.5 \pm 12.2$ \\
\hline $\begin{array}{l}\text { No taste } \\
\text { disturbance } \\
(n=296)\end{array}$ & $49.0 \pm 7.1$ & $49.2 \pm 10.2$ & $48.2 \pm 7.7$ & $43.6 \pm 8.9$ & $48.3 \pm 8.9$ & $49.2 \pm 10.3$ & $44.9 \pm 8.2$ & $48.1 \pm 9.0$ & $49.1 \pm 12.1$ \\
\hline $\mathrm{p}$-value & $<0.0001 * * * *$ & 0.089 & $0.007 * *$ & 0.869 & 0.199 & 0.149 & 0.085 & 0.684 & 0.207 \\
\hline $\begin{array}{l}\text { No halitosis } \\
(n=173)\end{array}$ & $48.8 \pm 7.4$ & $49.5 \pm 10.1$ & $48.7 \pm 8.3$ & $43.8 \pm 9.1$ & $48.4 \pm 8.6$ & $49.5 \pm 10.2$ & $44.6 \pm 8.4$ & $47.8 \pm 8.5$ & $48.7 \pm 10.9$ \\
\hline $\begin{array}{l}\text { Slight halitosis } \\
\quad(n=143)\end{array}$ & $50.2 \pm 7.6$ & $49.5 \pm 10.8$ & $48.3 \pm 7.6$ & $43.4 \pm 9.0$ & $48.3 \pm 9.2$ & $49.6 \pm 11.3$ & $45.7 \pm 8.8$ & $48.5 \pm 10.1$ & $50.0 \pm 13.5$ \\
\hline $\begin{array}{l}\text { Strong halitosis } \\
\qquad(n=2)\end{array}$ & $54.0 \pm 15.6$ & $43.0 \pm 5.7$ & $48.0 \pm 14.1$ & $42.0 \pm 1.4$ & $54.5 \pm 2.1$ & $37 \pm 0.0$ & $41.5 \pm 3.5$ & $54.0 \pm 1.4$ & $52.0 \pm 8.5$ \\
\hline $\mathrm{p}$-value & 0.179 & 0.681 & 0.880 & 0.890 & 0.624 & 0.256 & 0.418 & 0.519 & 0.597 \\
\hline
\end{tabular}

Hs, hypochondriasis; D, depression; Hy, hysteria; Pd, psychopathic deviation; Pa, paranoia; Pt, psychasthenia; Sc, schizophrenia; Ma, hypomania; Si, social introversion; MMPI-2, Minnesota Multiphasic Personality Inventory; RAU, recurrent aphthous ulceration; OLP, oral lichen planus.

Values are presented as mean \pm standard deviation.

p-values were completed by Student's t-test from thrush to taste disturbance. p-values were completed by one way ANOVA for halitosis.

${ }^{*} p<0.05,{ }^{* *} p<0.01, * * * * p<0.0001$.

significantly higher elevations on Sc clinical scale $(p<0.05)$. Subjects who have suffered OLP showed significantly higher mean values of T-scores than subjects who have not on Pa $(\mathrm{p}<0.05)$ and Ma $(\mathrm{p}<0.01)$ clinical scales. Mean values of T-scores on Ma clinical scale were significantly higher in subjects having experienced glossodynia than in subjects having not experienced $(\mathrm{p}<0.05)$. Subjects having felt taste disturbance exhibited significantly higher elevations on Hs $(p<0.0001)$ and Hy $(p<0.01)$ clinical scales compared to subjects having not (Fig. 1). No significant difference between subjects with a history of thrush and subjects without a history was demonstrated on all clinical scales. In addition, there was no significant difference on clinical scales according to the subjective degree of halitosis. 


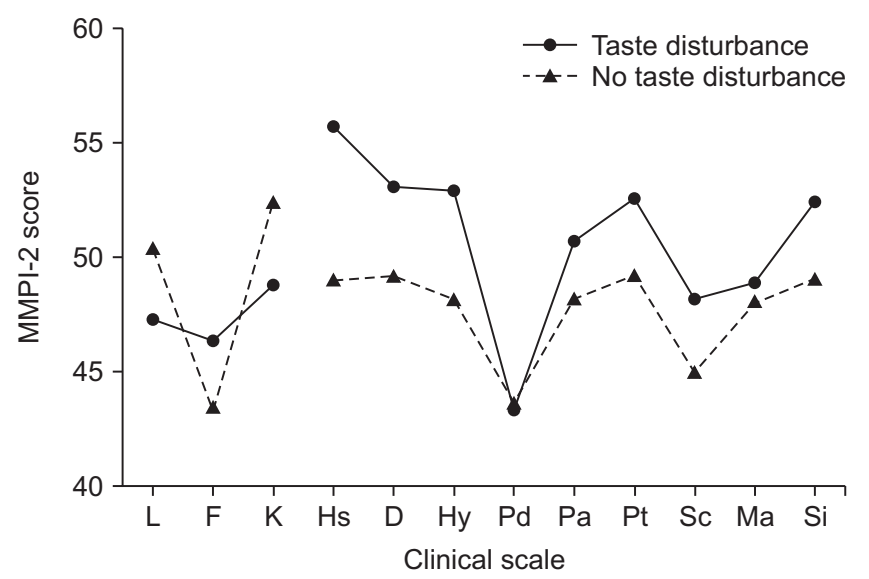

Fig. 1. The comparison of mean values of T-scores on Hs, D, Hy, $\mathrm{Pd}, \mathrm{Pa}, \mathrm{Pt}, \mathrm{Sc}, \mathrm{Ma}$, and $\mathrm{Si}$ clinical scales between subjects with a history of taste disturbance and subjects without a history. Three validity scales $(L, F$, and $K$ ) shows $V$ configuration, where the $L$ and $K$ values are high, and the $F$ scale value is low. MMPI-2, Minnesota Multiphasic Personality Inventory; L, lie; F, infrequency; $\mathrm{K}$, correction; Hs, hypochondriasis; $\mathrm{D}$, depression; Hy, hysteria; $\mathrm{Pd}$, psychopathic deviation; $\mathrm{Pa}$, paranoia; Pt, psychasthenia; Sc, schizophrenia; Ma, hypomania; Si, social introversion.

\section{DISCUSSION}

Oral mucosal conditions are accompanied by a high frequency of psychological problems [18]. it is generally assumed that psychosocial factors appear to be temporally related to the onset of recurrent herpes labialis [1]. The observation that episodes of recurrent herpes labialis in a student population are more frequent at examination periods suggests that environmental discomfort can make the occurrence of recurrent herpes labialis more probable [19]. The unhappy factor of the Clyde Mood Scale of nursing students was significantly correlated with the incidence of recurrent herpes labialis [6]. High scorers on Hs clinical scale of the MMPI-2 are generally dissatisfied and unhappy [20]. High scorers on Hy clinical scale of the MMPI-2 may report feeling sad, depressed, and anxious at times [20]. These findings coincides with the present result that subjects who have experienced herpetic stomatitis revealed significantly higher mean values of T-scores than subjects who have not on Hs and Hy clinical scales.

It is generally agreed that taste perception is intimately related to emotion in humans [21]. A distinct relationship between the resolution of dysgeusia and depression was identified [22]. Patients with sweet taste preference had significantly higher elevations on Hy clinical scale of the MMPI-2 [23]. Moderately high scores on Hs scale tend to be associated with diagnoses such as anxiety disorders and depressive disorders [20]. These studies support the current result that subjects having felt taste disturbance exhibited significantly higher elevations on Hs and Hy clinical scales compared to subjects having not.

Stress and anxiety have frequently been mentioned as possible factors related to the development of OLP, although this association is still debated [24]. Patients who manifest OLP have no greater tendency toward anxiety and no more stressful life events than other individuals assessing their stressful life events by Social Readjustment Rating Scale (SRRS) and their tendency for anxiety by the trait portion of the State-Trait Anxiety Inventory (STAI-S) [25]. There were no statistically significant associations between erosive OLP and either anxiety or depression, as measured on HADS [26]. Conversely, the patients with erosive lichen planus had significantly higher scores for the General Perceived Stress Questionnaire, Beck Depression Inventory (BDI) and STAI-S, indicating that depression, anxiety and stress is more common in patients with erosive lichen planus [27]. Bergdahl et al. [28] studied the psychologic aspects in 49 patients with oral lichenoid reactions and found that in the Karolinska Scales of Personality (KSP) they scored significantly hiqher in the muscular tension scale and the suspicion scale and in the Psychological Functioning Scale (PFS) had significantly more sad thoughts and became dizzy more easily and in the Personality Scale were significantly more worried, describing them as tense, suspicious, having sad thoughts, and being anxious. Moderately elevated scorers on Pa clinical scale of the MMPI-2 tend to be excessively sensitive and suspicious and may describe depression, sadness, and anxiety [20]. High scorers on Ma clinical scale of the MMPI2 may feel upset, tense, nervous, anxious and experience periodic episodes of depression [20]. In accordance with these findings the present result showed significantly higher mean values of T-scores of subjects who have suffered OLP on Pa and Ma clinical scales. In contrast, Ivanovski et al. [29] reported that no statistical differences between OLP and control groups were detected for the clinical scales of $\mathrm{Pd}$, $\mathrm{Pa}, \mathrm{Pt}, \mathrm{Sc}$, or Ma, whereas mean values of OLP subjects for Hs, D, and Hy scales were all significantly higher compared 
with control subjects.

Ott and Ott [30] pointed out that the sores of the selfrating anxiety and depression scales among the patients with glossodynia were higher than in healthy subjects, suggesting that glossodynia is an expression of a psychosomatic disorder. Trikkas et al. [10] demonstrated that introverted hostility, neuroticism and possibly depression are important components of the psychological profile of patients with glossodynia by means of Eysenck's Personality Questionnaire and Fould's Hostility and Direction of Hostility Questionnaire. The BMS patients were found to score significantly higher than the control group on most Symptom Checklist 90 subscales such as somatization, obsessive-compulsive, interpersonal sensitivity, depression, anxiety, hostility, phobic anxiety, and psychoticism [31]. A total of 72 BMS subjects in Toronto scored significantly higher than age- and sex-matched control subjects on Hs, D, Hy, Pd, Pa, Pt, Sc clinical scales of MMPI-2 [32]. On the contrary, Merigo et al. [33] found no significant differences in personality profiles using MMPI-2 between the twentyeight BMS and the 24 matched control subjects in Italy. In the current study, mean values of T-scores on Ma clinical scale were significantly higher in subjects having experienced glossodynia than in subjects having not experienced. Besides the present results showed that subjects who have experienced OLP or glossodynia scored significantly higher than subjects who have not experienced on Ma clinical scale in common. This finding is similar to an earlier study [28] in which it was reported that both the BMS patients and the oral lichenoid reaction patients scored high in the KSP muscular tension scale and became dizzy easily and had sad thoughts in the PFS, presenting with their similar personality profile.

A positive or negative association between RAU and patient's psychological status has been frequently described [34]. In HADS, 88\% of students with recurrent aphthous stomatitis (RAS) were having anxiety and 65\% of students with RAS had depression [34]. On the other hand, both sixty consecutive patients and 60 controls reported comparable depression and anxiety scores on HADS [35]. In one hundred and sixty subjects with a history of RAS, stressful life events were significantly associated with the onset of RAS episodes and mental stressors were more strongly associated with RAS episodes than physical stressors by means of the validated Recent Life Changes Questionnaire [36]. However, Pedersen [2] showed no association between psychologic life stress and recurrences of RAU in SRRS and Visual Analogue Scale scores. Using HADS, RAU patients reported higher levels of anxiety in comparison to controls, but both reported comparable depression scores, and NeuroticismExtraversionOpenness Five Factor Inventory scores among RAU patients were not different from those reported by controls [37]. Contrarily, there was statisticaly significant difference between thirty-nine RAS patients in Turkey and controls for depression using Hamilton's depression rating scale, while no statistical difference was found between the groups for both salivary cortisol levels and anxiety using Hamilton's anxiety rating scale and physiological testing instrument [38]. Gavic et al. [39] reported a high correlation between anxiety, depression, and psychological stress with symptoms of RAS administering BDI, STAI-S, and Ways of Coping Questionnaire to a total of 110 patients with RAS in the acute phase. To the contrary, comparison of stress, anxiety and depression between test group 1 (25 subjects) presenting with RAS and 25 normal subjects with no history of RAS, test group 2 (25 subjects) with a history of RAS but without oral ulcers and control group was found to be statistically insignificant by means of HADS and GHQ [40]. High scores on Sc clinical scale of the MMPI-2 indicate persons who may experience apprehension and generalized anxiety and feel insecure, blue, and depressed [20]. This disagrees with the current result that subjects without a history of RAU manifested significantly higher elevations on Sc scale than subjects with a history of RAU. This conflicting result may be attributable to the limitations of the present study including a diagnosis depending on the subjective self-reported symptom without an examination of the objective sign as well as a narrow age range of subjects.

In conclusion, symptoms of oral mucosal diseases such as herpetic stomatitis, taste disturbance, OLP, and glossodynia were found to be related to $\mathrm{Hs}, \mathrm{Hy}, \mathrm{Pa}$, Ma clinical scales of the MMPI-2. Therefore, comprehensive psychological evaluations of those oral mucosal diseases may be beneficial in a therapeutic approach. 


\section{CONFLICT OF INTEREST}

No potential conflict of interest relevant to this article was reported.

\section{ORCID}

Hye Sook Park

https://orcid.org/0000-0002-2981-3908

\section{REFERENCES}

1. Schmidt DD, Zyzanski S, Ellner J, Kumar ML, Arno J. Stress as a precipitating factor in subjects with recurrent herpes labialis. $\mathrm{J}$ Fam Pract 1985;20:359-366.

2. Pedersen A. Psychologic stress and recurrent aphthous ulceration. J Oral Pathol Med 1989;18:119-122.

3. Gallo CB, Mimura MAM, Sugaya NN. Psychological stress and recurrent aphthous stomatitis. Clinics (Sao Paulo) 2009;64:645648.

4. Cerqueira JDM, Moura JR, Arsati F, Lima-Arsati YBO, Bittencourt RA, Freitas VS. Psychological disorders and oral lichen planus: a systematic review. J Investig Clin Dent 2018;9:e12363.

5. Chaudhary S. Psychosocial stressors in oral lichen planus. Aust Dent J 2004;49:192-195.

6. Katcher AH, Brightman V, Luborsky L, Ship I. Prediction of the incidence of recurrent herpes labialis and systemic illness from psychological measurements. J Dent Res 1973;52:49-58.

7. Freire-Garabal M, Varela M, Riveiro P, et al. Effects of nefazodone on the immune system of mice. Eur Neuropsychopharmacol 2000;10:255-264.

8. Akpan A, Morgan R. Oral candidiasis. Postgrad Med J 2002;78: 455-459.

9. Guida RA. Candidiasis of the oropharynx and esophagus. Ear Nose Throat J 1988;67:832, 834-836, 838-840.

10. Trikkas G, Nikolatou O, Samara C, Bazopoulou-Kyrkanidou E, Rabavilas AD, Christodoulou GN. Glossodynia: personality characteristics and psychopathology. Psychother Psychosom 1996;65:163-168.

11. Gao J, Chen L, Zhou J, Peng J. A case-control study on etiological factors involved in patients with burning mouth syndrome. $\mathrm{J}$ Oral Pathol Med 2009;38:24-28.

12. Osaki T, Ohshima M, Tomita Y, Matsugi N, Nomura Y. Clinical and physiological investigations in patients with taste abnormality. J Oral Pathol Med 1996;25:38-43.

13. Schiffman SS. Taste and smell losses in normal aging and disease. JAMA 1997;278:1357-1362.

14. Bergdahl M, Bergdahl J. Perceived taste disturbance in adults: prevalence and association with oral and psychological factors and medication. Clin Oral Investig 2002;6:145-149.

15. Scully C, el-Maaytah M, Porter SR, Greenman J. Breath odor: etiopathogenesis, assessment and management. Eur J Oral Sci
1997; 105:287-293.

16. Pryse-Phillips W. An olfactory reference syndrome. Acta Psychiatr Scand 1971;47:484-509.

17. Oho T, Yoshida Y, Shimazaki Y, Yamashita Y, Koga T. Psychological condition of patients complaining of halitosis. J Dent 2001;29:31-33.

18. Tabolli S, Bergamo F, Alessandroni L, Di Pietro C, Sampogna F, Abeni D. Quality of life and psychological problems of patients with oral mucosal disease in dermatological practice. Dermatology 2009;218:314-320.

19. Ship II, Morris AL, Durocher RT, Burket LW. Recurrent aphthous ulcerations and recurrent herpes labialis in a professional school student population: III. Oral examinations. Oral Surg Oral Med Oral Pathol 1960;13:1438-1444.

20. Graham JR. MMPI-2: assessing personality and psychopathology. 5th ed. New York: Oxford University Press; 2011. pp. 63-221.

21. Nakagawa M, Mizuma K, Inui T. Changes in taste perception following mental or physical stress. Chem Senses 1996;21:195-200.

22. Deems DA, Yen DM, Kreshak A, Doty RL. Spontaneous resolution of dysgeusia. Arch Otolaryngol Head Neck Surg 1996;122:961963.

23. Aguayo GA, Vaillant MT, Arendt C, Bachim S, Pull CB. Taste preference and psychopathology. Bull Soc Sci Med Grand Duche Luxemb 2012;(2):7-14.

24. Vallejo MJ, Huerta G, Cerero R, Seoane JM. Anxiety and depression as risk factors for oral lichen planus. Dermatology 2001;203:303-307.

25. Allen CM, Beck FM, Rossie KM, Kaul TJ. Relation of stress and anxiety to oral lichen planus. Oral Surg Oral Med Oral Pathol 1986;61:44-46

26. McCartan BE. Psychological factors associated with oral lichen planus. J Oral Pathol Med 1995;24:273-275.

27. Lundqvist EN, Wahlin YB, Bergdahl M, Bergdahl J. Psychological health in patients with genital and oral erosive lichen planus. $\mathrm{J}$ Eur Acad Dermatol Venereol 2006;20:661-666.

28. Bergdahl J, Ostman P0, Anneroth G, Perris H, Skoglund A. Psychologic aspects of patients with oral lichenoid reactions. Acta Odontol Scand 1995;53:236-241.

29. Ivanovski K, Nakova M, Warburton G, et al. Psychological profile in oral lichen planus. J Clin Periodontol 2005;32:1034-1040.

30. Ott G, Ott C. Glossodynia--psychodynamic basis and results of psychopathometric investigations. J Psychosom Res 1992;36:677686.

31. Eli I, Kleinhauz M, Baht R, Littner M. Antecedents of burning mouth syndrome (glossodynia)--recent life events vs. psychopathologic aspects. J Dent Res 1994;73:567-572.

32. Grushka M, Sessle BJ, Miller R. Pain and personality profiles in burning mouth syndrome. Pain 1987;28:155-167.

33. Merigo E, Manfredi M, Zanetti MR, Miazza D, Pedrazzi G, Vescovi P. Burning mouth syndrome and personality profiles. Minerva Stomatol 2007;56:159-167.

34. Ajmal M, Ibrahim L, Mohammed N, Al-Qarni H. Prevalence and psychological stress in recurrent aphthous stomatitis among female dental students in Saudi Arabia. Clujul Med 2018;91:216221. 
35. Zwiri AM. Anxiety, depression and quality of life among patients with recurrent aphthous ulcers. J Contemp Dent Pract 2015;16:112-117.

36. Huling LB, Baccaglini L, Choquette L, Feinn RS, Lalla RV. Effect of stressful life events on the onset and duration of recurrent aphthous stomatitis. J Oral Pathol Med 2012;41:149-152.

37. Al-Omiri MK, Karasneh J, Lynch E. Psychological profiles in patients with recurrent aphthous ulcers. Int J Oral Maxillofac Surg 2012;41:384-388.

38. Polat C, Düzer S, Ayyıldız H, et al. Association between anxiety, depression, and salivary cortisol levels in patients with recurrent aphthous stomatitis. Turk Arch Otorhinolaryngol 2018;56:166169.

39. Gavic L, Cigic L, Biocina Lukenda D, Gruden V, Gruden Pokupec JS. The role of anxiety, depression, and psychological stress on the clinical status of recurrent aphthous stomatitis and oral lichen planus. J Oral Pathol Med 2014;43:410-417.

40. Dhopte A, Naidu G, Singh-Makkad R, Nagi R, Bagde H, Jain S. Psychometric analysis of stress, anxiety and depression in patients with Recurrent Aphthous Stomatitis-a cross-sectional survey based study. J Clin Exp Dent 2018;10:e1109-e1114. 
Appendix 1. Form and contents of questionnaire

\section{Questionnaire}

Name of College: $\quad$ Name of Department: Grade:

Name: Gender:

Please answer the following questions by checking the one answer which describes you.

1. Have you ever had white or grayish patches on oral mucosa like tongue, palate or inner cheeks?
1) Yes
2) No

2. Have you ever suffered blisters followed by ulcers on the lips or in the mouth?
1) Yes
2) No

3. Have you ever experienced painful ulcers which are round, shallow, yellowish-gray colored on tongue, inner cheeks, or lips?
1) Yes
2) No

4. Have you ever had an widely excoriated surface which is sore on inner cheeks or gingiva?
1) Yes
2) No

5. Have you ever felt the pain or unusual sensation in the anterior portion of the tongue?
1) Yes
2) No

6. Have you ever felt altered, diminished, or unpleasant sense of taste of food?
1) Yes
2) No

7. Do you feel your oral malodor?
1) No
2) Slightly feel
3) Strongly feel

Thank you for your cooperation. 